\title{
Prokaryotic expression, purification of a novel candidate tumor suppressor gene FUS1 and characterization of its polyclonal antibodies
}

\author{
Dong-Mei Zhang, Han-Shuo Yang, Xin-Yu Zhao, Wen Zhu, Zhi-Hua Feng, Yang Wan, Zhi-Wei Zhao, \\ Ming-Hai Tang, Nong-Yu Huang, Yu-Quan Wei
}

State Key Laboratory of Biotherapy, West China Hospital, West China Medical School and School of Life Sciences, Sichuan University, Chengdu, China.

Email: yuquawei@vip.sina.com; zwjulia@163.com

Received 6 June 2009; revised 27 November 2009; accepted 4 December 2009.

\begin{abstract}
FUS1 is a novel candidate tumor suppressor gene identified in human chromosome 3p21.3. Its expression showed significantly reduction or even loss in lung cancer and other types of cancers. In order to further investigate the biological function of FUS1 protein, FUS1 cDNA from MRC-5 cells was amplified by RT-PCR and cloned into prokaryotic expression vector $P Q E-30$. The recombinant expression plasmids were transformed into M15 strain and grown at $20^{\circ} \mathrm{C}$ or $37^{\circ} \mathrm{C}$. SDS-PAGE analysis revealed that the accumulation of the recombinant protein FUS1 (rFUS1) in inclusion body forms reached maxium amount when induced with $0.5 \mathrm{mM}$ IPTG for $5 \mathrm{~h}$ at $37^{\circ} \mathrm{C}$. The inclusion bodies were solubilized in $2 \mathrm{M}$ urea and purified by a 6 × His tagged affinity column under denaturing condition. The purified rFUS1 was identified by electrospray ionization-mass spectrometry (ESI-MS) and tested for purity by HPLC chromatography. The purified rFUS1 proteins were then used to immunize rabbits to obtain anti-human FUS1 polyclonal antibodies, which were suitable to detect both the recombinant exogenous FUS1 and the endogenous FUS1 from tissues and cells by western blot and immunohistochemistry, Available purified rFUS1 proteins and self-prepared polyclonal antibodies against FUS1 may provide effective tools for further studies on biological function and application of FUS1.
\end{abstract}

Keywords: FUS1; Polyclonal Antibody; Prokaryotic Expression; Recombinant Protein; Tumor Suppressor Gene

\section{INTRODUCTION}

With a rapidly increasing incidence and a low cure rate, lung cancer has been the most common malignancy and leading cause of cancer deaths in the world [1]. Therefore, identification of new therapeutic targets and novel strategies are essential to improve the survival of patients diagnosed with this disease. FUS1 is a novel tumor-suppressor gene located on the human chromosome 3p21.3 region [2]. Genetic alterations and allelic loss of 3p21.3 are among the most frequent and earliest cancer abnormalities detected in the pathogenesis of lung cancers. This phenomenon occurs in almost $100 \%$ of small cell lung cancers (SCLCs) and more than $80 \%$ of non-small-cell lung cancers (NSCLCs) [3]. FUS1 functions as "gatekeepers" of human cancer and plays a very important role in lung cancer development $[4,5]$. A majority of human lung cancers have been found loss of expression, haploinsufficiency or deficiency of posttranslational of FUS1 $[2,6,7]$. FUS1 was recently demonstrated to mediate accumulation of p53 protein, and thus led to the apoptosis and cell cycle arrest in the early stages of lung cancer progression [8,9].However, many properties of FUS1 biological functions as well as its action mechanism are not well understood and little is known about its basic structure-function relationships. Therefore, the expression and the preparation of recombinant protein FUS1 and its polyclonal antibody should provide effective experimental tools for further identifying its mechanism and biological functions against cancers.

In the present study, the full length FUS1 with six histidine ( $6 \times$ His) residues was expressed in Escherichia coli M15. The recombinant protein was purified by urea and identified by electrospray ionization-mass spectrometry analysis (ESI-MS). The polyclonal antibodies against FUS1 were prepared which were suitable to detect the presence of both the exogenous and endogenous FUS1 efficiently by western blot and immunohistochemistry. The self-prepared polyclonal antibodies may be applied as the effective tools for investigating the further molecular mechanisms of FUS1. 


\section{MATERIALS AND METHODS}

\subsection{Materials}

Escherichia coli strain JM109 and M15 were maintained by our Lab. The human non-small-cell lung cancer (NSCLC) cell line A549 and normal human lung fibroblast cell line MRC-5 were obtained from ATCC and were maintained in RPMI 1640 or DMEM supplemented with $10 \%$ FCS respectively. pQE-30 plasmid was purchased from Qiagen (Germany). Human paracancerous normal lung tissues and lung cancer tissues were obtained from Department of Thoracic and Cardiovascular Surgery, West China Hospital, Sichuan University. Restriction endonucleases, T4 DNA ligase and prestained low range protein molecular weight marker were products of MBI Fermentas (Lithuania). The High Fidelity PrimeScript ${ }^{\mathrm{TM}}$ RT-PCR Kit was purchased from TaKaRa (Dalian, China). All PCR products used for cloning were confirmed by sequencing at Invitrogen Biotechnology Co., Ltd (Shanghai, China). pVITRO2-FUS1 plasmid was constructed by our Lab.

\subsection{Total RNA Isolation}

Total RNA was isolated from MRC-5 cells using a standard Trizol RNA isolation protocol. Prior to use, RNA concentration was spectrophotometrically determined, and RNA integrity was verified by electrophoresis.

\subsection{RT-PCR Amplification of FUS1 cDNA}

The cDNA of full length FUS1 was prepared using a High Fidelity PrimeScript ${ }^{\mathrm{TM}}$ RT-PCR Kit according to the manufacturer instructions. Based on the FUS1 cDNA sequence (GenBank accession No. AF055479), a pair of primers was designed as follows:

5'-CGCGGATCCATGGGCGCCAGCGGGTCCAAAG3'(sense) and 5'-CCGGTCGACTCACACCTCATAGAG GATCACAG-3'(anti-sense). The sense and anti-sense primers were introduced $\mathrm{BamHI}$ and SalI restriction sites (underlined) respectively.

\subsection{Plasmid Construction and Identification}

The amplified FUS1 cDNA and expression vector pQE-30 were digested with restriction enzymes BamHI/SalI respectively. The digested products were separated on a $1 \%$ agarose gel and the bands were extracted. The purified FUS1 cDNA and the linear vector were ligated overnight at $16^{\circ} \mathrm{C}$ with T4 DNA ligase followed by transformed into E.coli JM109 competent cells. The transformation mixture was plated on to LB agar plates containing ampicillin $(100 \mu \mathrm{g} / \mathrm{ml})$. The plates were incubated for $16 \mathrm{~h}$ at $37^{\circ} \mathrm{C}$. The desired recombinant plasmid pQE-30-FUS1 was confirmed by PCR and restriction enzyme digestion with BamHI/SalI and DNA sequencing (Invitrogen).

\subsection{Expression of Fusion Proteins}

E. coli M15 cells were transformed with recombinant plasmid pQE-30-FUS1 extracted from JM 109. The bacteria cells were cultured at $37^{\circ} \mathrm{C}$ in $5 \mathrm{ml} \mathrm{LB}$ liquid medium with $100 \mu \mathrm{g} / \mathrm{ml}$ ampicillin for 4-6 h at $220 \mathrm{rpm}$ till to an absorbance of 0.6 at $600 \mathrm{~nm}$. Expression of the fusion protein was induced by isopropyl- $\beta$-D-thiogalactopyranoside (IPTG) with a final concentration of $0.5 \mathrm{mM}$ for $5 \mathrm{~h}$ at $37^{\circ} \mathrm{C}$. A time and temperature course of expression and solubility to determine the optimal induction conditions for maximum expression of protein was measured by taking aliquots of cells at $1,2,3,4$ and $5 \mathrm{~h}$ after induction with IPTG at $37^{\circ} \mathrm{C}$ and $20^{\circ} \mathrm{C}$ These samples were harvested by centrifugation at $13,000 \mathrm{rpm}$ for $1 \mathrm{~min}$. The pellets were resuspended in PBS ( $\mathrm{pH} 8.0$ ) and sonicated for $6 \times 10 \mathrm{~s}$ with $10 \mathrm{~s}$ pauses at $20 \mathrm{~W}$ on ice. The total lysate induced with IPTG was divided into soluble and insoluble fractions by centrifugation at 15,000 rpm for $2 \mathrm{~min}$ at $4{ }^{\circ} \mathrm{C}$. The expression and solubility of FUS1 protein were then analyzed in parallel by 15\% SDS-PAGE followed by staining with Coomassie Brilliant Blue R-250. The protein extracts of cells transformed with the uninduced bacteria were used as the control.

\subsection{Extraction of Fusion Proteins}

For large scale expression and purification, M15 strain was transformed with the plasmid and cultured in $1 \mathrm{~L}$ of $\mathrm{LB}$ medium at $37^{\circ} \mathrm{C}$ until to an absorbance of 0.6 at $600 \mathrm{~nm}$ followed by induction of $0.5 \mathrm{mM}$ IPTG at $37^{\circ} \mathrm{C}$ for an additional $5 \mathrm{~h}$ before harvested. The cells were harvested by centrifugation at 4,000 rpm for $20 \mathrm{~min}$ at $4{ }^{\circ} \mathrm{C}$. The pellet was resuspended in PBS (20mM PB, $500 \mathrm{mM} \mathrm{NaCl}, \mathrm{pH}$ 8.0). Extraction was performed using a French Pressure Cell Press (APU Co., 04010008) at an internal pressure of 800 psi [10]. The remaining pellet was then harvested by centrifugation at 15,000 rpm for $30 \mathrm{~min}$ at $4^{\circ} \mathrm{C}$. The supernatant (soluble fraction) was collected for analysis later. The inclusion bodies were weighted and solution buffer (20 mM PB, $500 \mathrm{mM} \mathrm{NaCl}$, $2 \mathrm{M}$ urea, $\mathrm{Ph}$ 8.0) were pulled to suspend the cells, then the supernatant were collected by centrifugation at $15000 \mathrm{rpm}$ for $30 \mathrm{~min}$ at $4^{\circ} \mathrm{C}$. The purified recombinant protein was confirmed by SDS-PAGE and Western blotting using anti-His monoclonal antibody conjugate to HRP. The concentration of the protein was determined according to Bradford [11].

\section{7. rFUS1 Identification by ESI-MS Analysis}

The presence of purified rFUS1 in the eluted fractions was separated on 15\% SDS-PAGE and identified by electrospray ionization (ESI)-mass spectrometry (MS). The gel band stained with Coomassie brilliant blue R-250 was excised minced, reduced, alkylated with io- 
doacetamide, In-gel digestion of proteins was carried out with $12.5 \mathrm{ng} / \mu \mathrm{l}$ mass spectrometry grade Trypsin Gold (Promega) for $12-16 \mathrm{~h}$ at $37^{\circ} \mathrm{C}$ [12]. The tryptic peptides were extracted twice with the buffer containing $50 \%$ ACN $/ 0.1 \%$ TFA for $15 \mathrm{~min}$, and the solutions were combined together. Mass spectra were acquired using an ESI-Q-TOF mass spectrometer (Micromass, Manchester, UK) with $15 \mu$ l of tryptic peptides solution. The MS/MS data were acquired by the software of MassLynx (Micromass) and converted to PKL files by the software of ProteinLynx 2.2.5 (Waters) were then analyzed using MASCOT search engine (http://www.matrixscience. com).

\subsection{Preparation, Purification of rFUS1 Poly- clonal Antibodies}

The purified rFUS1 was used to prepare antibodies in New Zealand white rabbit. The rabbit was first immunized subcutaneously with rFUS1 (200 $\mu$ g) in complete Freund's adjuvant. Two booster injections were given in incomplete Freund's adjuvant every week. The serum was collected 7 days after the 3rd immunization to determine the antibody titer by enzymelinked immunosorbent analysis (ELISA). The last immunization was performed one week later, and the antiserum was collected through heart after 7 days. The rabbit IgG fraction was precipitated from the immune serum with $50 \%$ saturated $\left(\mathrm{NH}_{4}\right)_{2} \mathrm{SO}_{4}$ and purified by DEAE-Sepharose column chromatography.

\subsection{Specificity Analysis of the Polyclonal Antibodies by Western Blot}

The specificity of the antiserum was tested by western blot analysis using the total proteins of MRC-5 cells and those of A549 cells transfected with FUS1 constructs. rFUS1 and the total proteins of A549 cells untransfected were used as control. The cells were harvested and lysed in lysis buffer [10 mM Tris/HCl, pH 8.0, $150 \mathrm{mM} \mathrm{NaCl}$, $1 \%$ Triton X-100 and $1 \mathrm{mM}$ DTT supplemented with protease and phosphatase inhibitors (2 $\mathrm{mM}$ sodium orthovanadate, $100 \mathrm{nM}$ okadaic acid, $1 \mathrm{mM} \mathrm{NaF}, 1 \mathrm{mM}$ $\beta$-glycerophosphate and cocktail (Sigma)] according to the related methods [13]. The protein samples were separated on 15\% SDS-PAGE and electrophoretically transferred onto PVDF (polyvinyllidenefluoride) membrane. After blocking overnight in 5\% (w/v) non-fat milk, the PVDF membranes were incubated with serum at a dilution of 1:1000 for $2 \mathrm{~h}$. The membranes were washed three times with TBST buffer and then incubated in goat anti-rabbit IgG conjugated with HRP at a dilution of 1:10000 for $1 \mathrm{~h}$ at $37^{\circ} \mathrm{C}$.After washing two times with TBST buffer, one time with TBS then analyzed using the enhanced chemiluminescence detection system and exposed to Kodak BioMax X-ray film for 2-5 min.

\subsection{Cell and Tissue Immunohistochemistry}

In order to further confirm that the polyclonal antibodies against rFUS1 are suitable for application in recognizing the innate FUS1 proteins from cells or tissues, immunohistochemistry was performed in A549 cells transfected with FUS1 constructs and normal lung tissue respectively. A549 cells were seeded onto coverslips and cultured in RPMI-1640 with $10 \%(\mathrm{v} / \mathrm{v})$ FBS (fetal bovine serum) at $37^{\circ} \mathrm{C}$ in $5 \% \mathrm{CO} 2$. The cells were transfected with recombinant plasmid pVITRO2-FUS1 and vector pVITRO2 when cell confluence reached $60 \%$. Hoechst 33258 staining was used to identify apoptosis induced by FUS1 expression in A549 cells. At 48 h post-transfection, cells were fixed with fresh Carnoy's fixative, stained with Hoechst 33258 for $30 \mathrm{~min}$ at the concentration of $0.5 \mu \mathrm{g} / \mathrm{ml}$. Stained nuclei were detected after washing twice with distilled water and observed under a fluorescence microscope. At the same time, the coverslips transfected with pVITRO2-FUS1 was immersed in icecold acetone to fix for $20 \mathrm{~min}$ on ice. The cell was permeabilized with $0.2 \%$ Triton $\mathrm{X}-100$ for 10 min after washing two times with deionized water, the slips were blocked with goat serum albumin at $37^{\circ} \mathrm{C}$ for $15 \mathrm{~min}$, the anti-rFUS1 polyclonal serum was used as the primary antibody (1:750). The second antibody was a biotinylated goat anti-rabbit IgG. The cells were then stained with HRP-streptavidin reagents (Dako) and DAB. Brown staining was considered positive.

As for tissue immunohistochemical analysis, normal lung tissues were fixed in $10 \%$ buffered neutral formalin and embedded in paraffin. Then, indirect immunostaining for FUS1 was performed on paraffin-embedded tissues by using the LSAB (labelled streptavidin-biotin) method to visualize antibody response as described above.

\section{RESULTS AND DISCUSSION}

\subsection{Construction and Identification of Expression Plasmid pQE-30-FUS1}

For amplification of FUS1 cDNA, RT-PCR was performed with total RNA from MRC-5 cell as the template, using gene-specific primers containing a $B a m \mathrm{H}$ I site or a $\mathrm{Sal}$ I site to facilitate cloning into expression plasmid pQE-30. A DNA fragment, approximate 350 bp in length, was obtained as shown (Figure 1(a)), which is consistent with the FUS1 cDNA 333 bp in length.

The amplified FUS1 cDNA was inserted into the sites of $\mathrm{BamH}$ I and $\mathrm{Sal}$ I in the expression plasmid pQE-30. The recombinant plasmid PQE-30-FUS1 was verified by PCR using FUS1-specific primer and restriction endonuclease digestion with $\mathrm{BamH} \mathrm{I} / \mathrm{Sal}$ I (Figure 1(b)) and DNA sequencing (data not shown). 


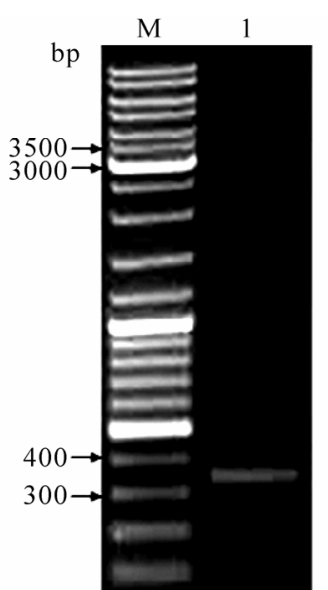

(a)

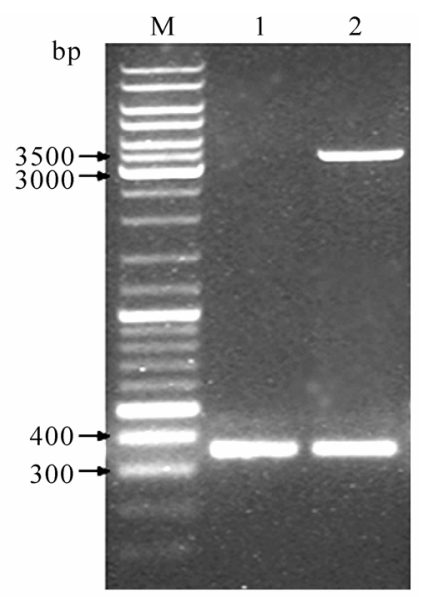

(b)
Figure 1. Cloning of FUS1 cDNA and identification of recombinant plasmid pQE-30-FUS1. (a) FUS1 cDNA was amplified from the total RNA of MRC-5 cell by RT-PCR. M: DNA marker; lane 1: FUS1 cDNA fragment. (b) Recombinant plasmid pQE-30-FUS1 was identified by PCR and BamH I/Sal I digestion. M: DNA marker; lane 1: FUS1 cDNA fragment obtained by PCR using FUS1-specific primers with recombinant plasmid as template; lane 2: DNA fragments obtained by Bam H I/Sal I digestion.

\subsection{Expression and Solubility Identification of pQE-30-FUS1}

The rFUS1 was produced in $E$. coli M15 as a fusion protein with $6 \times \mathrm{His}$ tag at the $\mathrm{N}$-terminus. The expressed protein was approximate $16 \mathrm{kDa}$. The $E$. coli cells containing recombinant plasmid pQE-30-FUS1 were cultured in $5 \mathrm{ml}$ of LB medium by adding IPTG at a final concentration of $0.5 \mathrm{mM}$. The solubility of FUS1 protein was examined by varying induction temperature from $20^{\circ} \mathrm{C}$ to $37^{\circ} \mathrm{C}$. No observable difference was observed in the expression form of the rFUS1. SDS-PAGE analysis revealed that the expression of FUS1 in E.coli was mainly in insoluble form (Figure 2). In addition, we also constructed recombinant plasmid pET-32a (+) -FUS1. When it was expressed in E. coli, no significant difference in the solubility of the rFUS1 was observed, except that there was more hybridprotein in inclusion bodies which enhanced the difficulty of the purify of recombinant protein than plasmid pQE-30-FUS1 (data not shown). Therefore, in the present study, FUS1 expression from pQE-30-FUS1 was induced with $0.5 \mathrm{mM}$ IPTG for $5 \mathrm{~h}$ at $37^{\circ} \mathrm{C}$ for further experiments.

In order to determine the optimal induction time for maximum expression of the protein, the cells were incubated for 1 to $5 \mathrm{~h}$ after IPTG was added. The rFUS1 protein showed expression at $1 \mathrm{~h}$ post-induction and the maximum protein amount could be achieved at the fifth hour at $37^{\circ} \mathrm{C}$ (Figure 2(a)), while its synthesis rate was low at $20^{\circ} \mathrm{C}$ (Figure 2(b)).

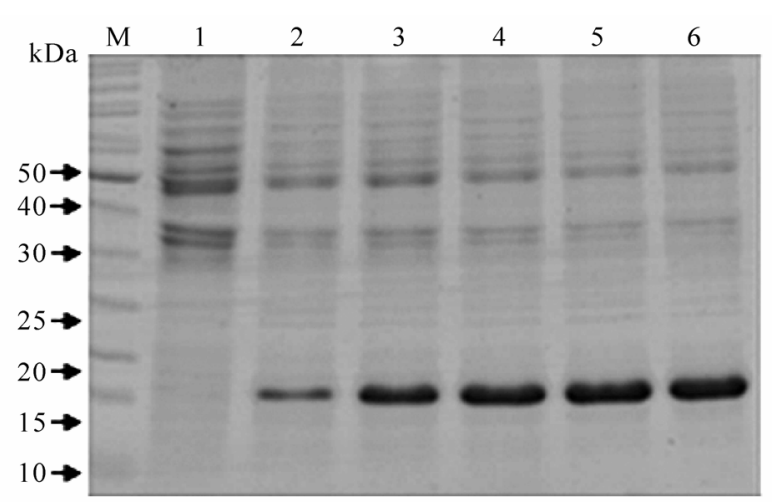

(a)

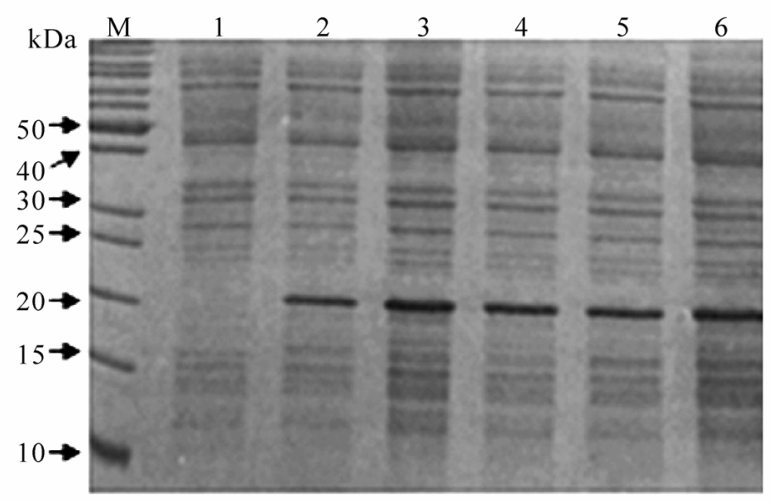

(b)

Figure 2. SDS-PAGE analysis of recombinant protein FUS1 produced in $E$. coli M15 by induced with $0.5 \mathrm{mM}$ IPTG at $37^{\circ} \mathrm{C}$ (a) and $20^{\circ} \mathrm{C}$ (b) from 1 to 5 hours. The cell lysates were analyzed every one hour after IPTG induction. M: protein molecular marker; lane 1: total bacterial protein without IPTG induction; lane 2-6: total bacterial protein at the established time ends.

\subsection{Purification of rFUS1}

$15 \%$ SDS-PAGE analysis revealed that the expression of rFUS1 in E. coli M15 was nearly $100 \%$ in insoluble forms at $37^{\circ} \mathrm{C}$ for $5 \mathrm{~h}$ (Figure 3(a)). For purification of the recombinant protein FUS1, cells collected from 1 liter LB culture were pressured by a French Pressure Cell Press. The inclusion bodies were harvested by centrifugation at $15,000 \mathrm{rpm}$ for $30 \mathrm{~min}$ at $4^{\circ} \mathrm{C}$. The pellet was washed by gradient urea from $1 \mathrm{M}$ to $8 \mathrm{M}$ [14]. The inclusion bodies began to dissolve in $2 \mathrm{M}$ urea buffer. More rFUS1 were dissolved to a gradually increasing concentration of urea with more hybrid proteins. So the inclusion bodies were dissolved in $2 \mathrm{M}$ urea buffer. After purification, rFUS1 were analyzed by $15 \%$ SDS-PAGE and showed a single band at the expected molecular mass (16 kD) on SDS-PAGE (Figure $3(\mathbf{b})$ ). The purity of the rFUS1 protein was proved to be higher than $90 \%$ by HPLC chromatography (data not shown) and the protein concentration was determined using Bradford reagent. 


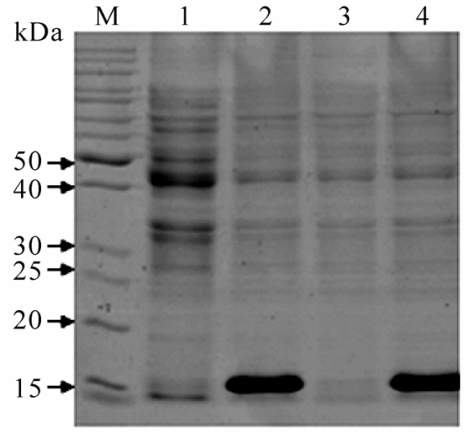

(a)

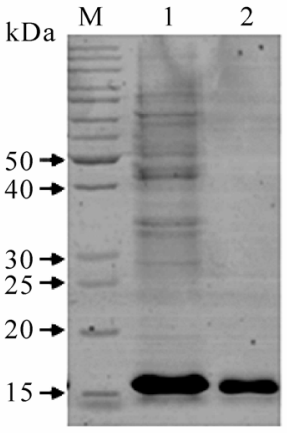

(b)
Figure 3. Solubility identification and purification of rFUS1 in E. coli M15. (a) the M15 cells were cultured at $37^{\circ} \mathrm{C}$, induced with $0.5 \mathrm{mM}$ IPTG for $5 \mathrm{~h}$ and assayed by SDS-PAGE analysis. Lane 1: total bacterial protein without IPTG induction; lane 2: total bacterial protein with IPTG induction; lane 3: supernatant with IPTG induction; lane 4: precipitate with IPTG induction; (b) Purification of rFUS1.Lane 1: The precipitate of total bacterial protein containing rFUS1 with IPTG induction. Lane 2: purified rFUS1; M: protein molecular marker.

\subsection{Western Blot Anlysis of the Purified rFUS1}

After SDS-PAGE, rFUS1 was transferred to a nitrocellulose membrane. The membrane was incubated with blocking buffer $[0.1 \%$ Tween and $5 \%$ non-fat milk in Tris-buffered saline (TBS)] overnight at $4^{\circ} \mathrm{C}$ and then with the anti-His-tag monoclonal antibody conjugate HRP diluted 1:1000. The membrane was washed 3 times with washing solution (Tween $0.1 \%$ in TBS) and incubated with an antimouse peroxidase-conjugated antibody (KPL) diluted 1:5000. After washed 3 times with washing solution, the membrane was treated with SuperSignal West Pico Chemiluminescent Substrate (Pierce) for $5 \mathrm{~min}$, then exposed to HyperWlm (Amersham Biosciences) for 5 min and visualized. The purified rFUS1 was verified successfully using Western-blot analysis through antiHis-tag mAb (Figure 4).

\section{5. rFUS1 Identification by ESI-MS Analysis}

The purified recombinant FUS1 proteins were further validated by ESI-MS analysis. The result from MS data suggested that the identified protein exactly matched with human FUS1 protein, which had a Mascot score 1018. MS/MS analysis revealed that seven unique peptide unambiguously matched to the target FUS1 protein (Figure 5(a)). For example, MS/MS spectrum of parent ion 861.7737 and the result of peptide sequence query were shown in Figure 5(b). All matched peptides were shown in Figure 5(c) (underlined), which indicated that the purified recombinant protein FUS1 were completely correct. A sufficient amount of purified FUS1 protein would make it possible to prepare polyclonal antibodies against FUS1 and to further analyze its interacting proteins or structure by X-ray crystallography.

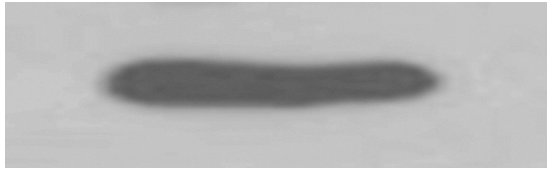

Figure 4. Analysis of purified rFUS1 by Western blot. The purified recombinant protein FUS1 was separated on 15\% SDSPAGE and probed by with anti-His tag mAb conjugated with HRP (1:10000). Chemiluminescence immunoassay was used for color development.
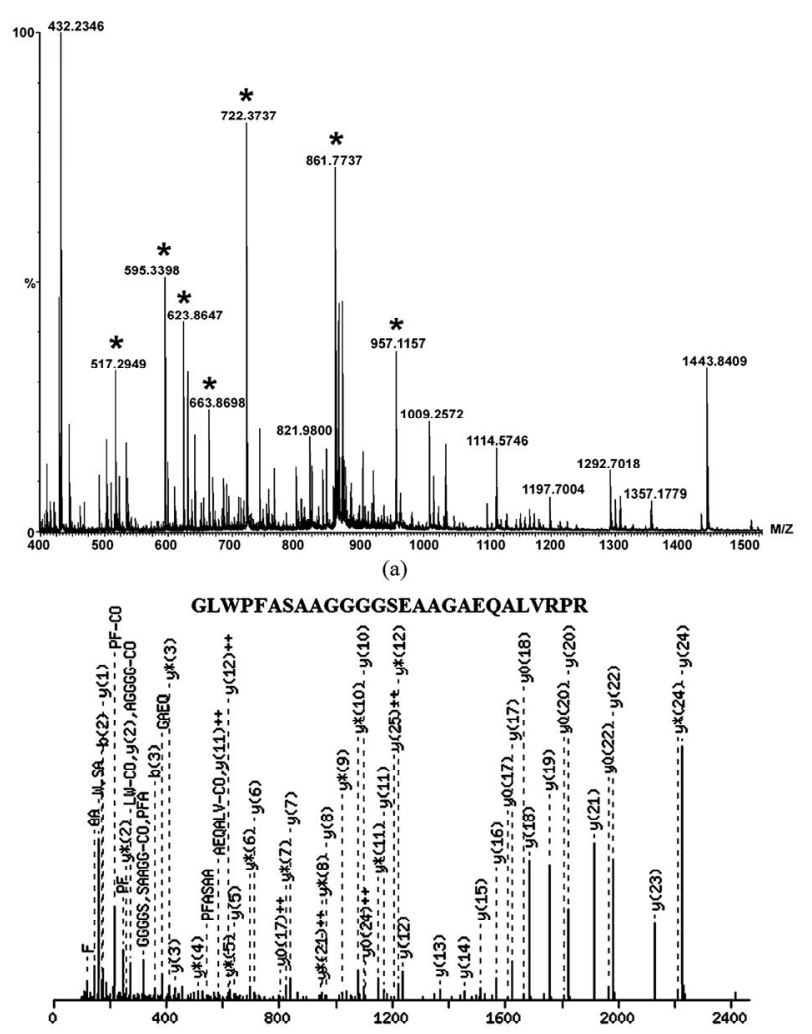

(b)

MGASGSKARG LWPFASAAGG GGSEAAGAEQ ALVRPRGRAV PPEVFTRRGS MFYDEDGDIA HEFYEETIVT KNGQKRAKLR RVHKNLIPQG IVKLDHPRIH VDFPVILYEV

(c)

Figure 5. ESI-MS/MS identification of tryptic peptides from the purified recombinant protein. (a) mass spectrogram of tryptic peptides from purified FUS1 protein. Totally 7 unique peptides were matched to target protein; (b) MS/MS spectrum of parent ion 861.7737 as an example. Data base search indicated its peptide sequence was GLWPFASAAGGGGSEAAGAEQALVRPR, which was a part of the sequence of FUS1; (c) matched peptides (underlined).

\subsection{Characteristics of Antiserum Against FUS1}

The specificity of the antiserum from rabbit against purified rFUS1 and total proteins extracted from MRC-5 was checked by Western blot analysis. FUS1 has been proved no protein level expression in A549 cells though FUS1 
mRNA was detectable [3,6]. Therefore, total proteins from A549 cells transfected with expression plasmid pVITRO2-FUS1 was also used in Western blot. The result indicated that the antiserum from rabbit can recognize both exogenous recombinant FUS1 and endogenous FUS1 effectively. There were positive bands at the position of approximately $16 \mathrm{kDa}$ (Figure 6). However, nonimmunized serum was negative (data not shown).

The FUS1 protein is proved to be indeed present in the cytoplasm and may have a role in signal transduction [8]. In the present study, FUS1-negative A549 cells were stained with Hoechst 33258 after $24 \mathrm{~h}$ transient transfection with pVITRO2-FUS1. More hypercondensed nuclei and apoptotic bodies appeared in the transfected A549 cells (brightly stained; Figure 7(c)) comparing with that in the control cells (Figure 7(a) and (b)). The result of FUS1 inducing apoptosis consisted with the findings reported previously by Ji et al. and Ito et al. $[8,15]$ FUS1-mediated apoptosis is proved to be associated with the accumulation of p53 protein, the down-regulating expression of MDM2 and the activation of Apaf1-drivened mitochondrial apoptotic pathway [9]. However, the exact mechanism of inactivation of FUS1 in human tumorigenesis and its role in FUS1-mediated tumor suppression are still unclear and need to be investigated in detail.

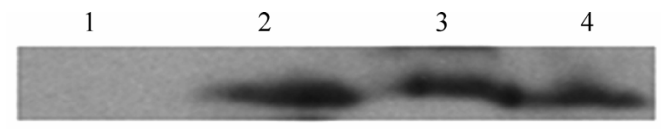

(a)

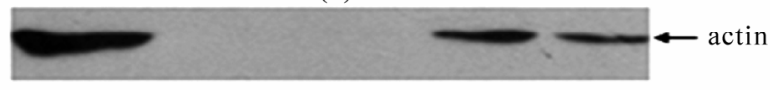

(b)

Figure 6. Western-blot analysis of FUS1 expression using the rabbit antiserum (1:1000). (a) the total protein from A549 (lane 1), purified rFUS1 (lane 2), the total protein from MRC-5 cells (lane 3) and the total protein from A549 cells after transient transfection with FUS1 constructs (line 4) were loaded. Horse radish peroxidase-conjugated goat anti-rabbit IgG (1:10000) and enchanced chemiluminescence were used for color development; (b) the same blots were probed by $\beta$-actin as control.

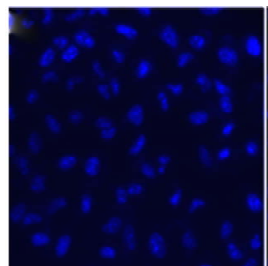

(a)

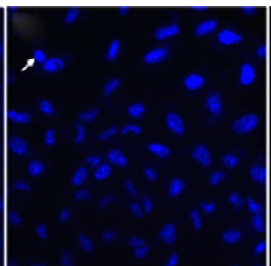

(b)

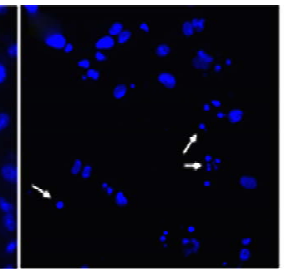

(c)
Figure 7. Induction of apoptosis in A549 cells by the expression of FUS1. (a) the untreated A549 cells as negative control; (b) A549 cells transfected with pVITRO2; (c) A549 cells transfected with pVITRO2-FUS1. At 24 h post-transfection, the cells were stained with Hoechst 33258. The arrows indicated the representatives of apoptotic cell. Original magnification, $\times 200$.
To further elucidate the relationship between FUS1 expression and apoptosis, the self-prepared anti-rFUS1 polyclonal antibodies were used for immunostaining in A549 cells transfected with pVITRO2-FUS1 and untreated cells. At the same time, immunohistochemistry was also performed in paracancerous normal lung tissues and lung cancer tissues. There were strong brown staining in the cytoplasm of A549 cells transfected with FUS1 constructs and that of paracancerous normal lung tissues (Figure 8(a) and (c)). However, no immunostaining was observed in the cytoplasm of non-transfected A549 cells and that of lung cancer tissues (Figure 8(b) and (d)). These results are consistent with previous findings and provide further evidence that FUS1 plays important roles in tumor-suppression function and lung cancer development. The similar results were obtained in liver, stomach,

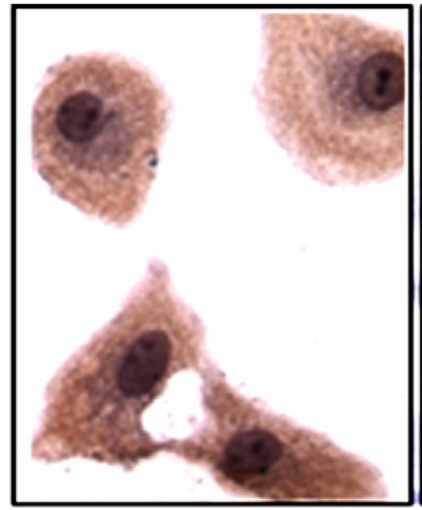

(a)

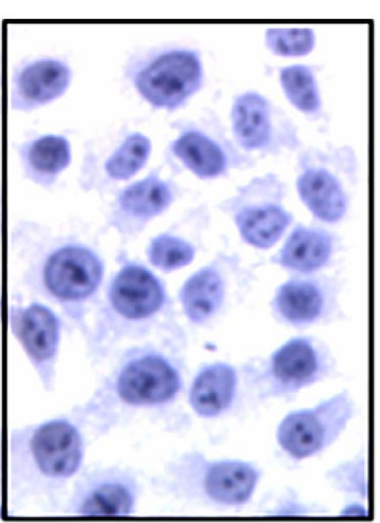

(b)

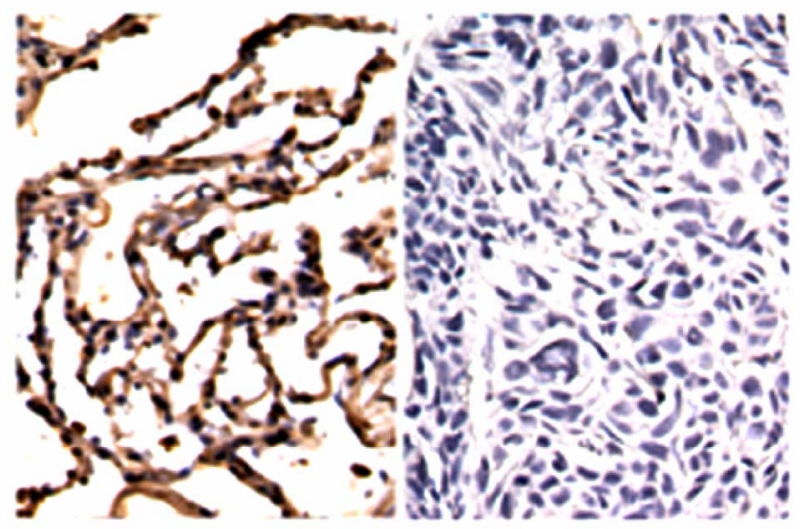

(c)

(d)

Figure 8. Analysis of FUS1 expression using the self-prepared rabbit anti-human rFUS1 polyclonal antibodies (1:750). Immunostaining was performed in A549 cells transfected with pVITRO2-FUS1 or not (a and b) and paracancerous normal lung tissues and lung cancer tissues (c and d). The anti-FUS1 polyclonal antibodies (1:750) can recognize the FUS1 protein in the cytoplasm of A549 cells transfected with FUS1 constructs and that of paracancerous normal lung tissues (a and c) comparing with the corresponding controls (b and d). Brown staining showed the positive results. Haematoxylin staining showed the cell nuclei. Original magnification, $\times 200$. 
cervix, endometrial and ovarian carcinomas when using the self-prepared anti-FUS1 polyclonal antibodies (data not shown).

\section{CONCLUSIONS}

FUS1 is a novel tumor-suppressor gene located on human chromosome 3p21.3 that is frequently deleted in human lung and breast cancers. But there is no commercial antibody against full-length FUS1 until now. In the present work, human full-length FUS1 cDNA was cloned and expressed as a fusion protein with six histidine $(6 \times$ His) tag in $E$. coli. The purified recombinant protein was identified by ESI-MS (electrospray ionization MS) analysis. Furthermore, the specific and sensitive polyclonal antibodies against full-length FUS1 were raised, which were suitable to detect both the recombinant exogenous FUS1 and the endogenous FUS1 from tissues and cells by western blot and immunohistochemistry. To our knowledge, this is the first report of soluble expression and purification of rFUS1 protein and generation of antifull-length FUS1 polyclonal antibodies so far. The purified rFUS1 proteins and self-prepared polyclonal antibodies against FUS1 may provide effective tools for further studies on biological function and mechanism of FUS1 in pathogenesis of lung and other carcinomas in the future.

FUS1 protein expression rarely existed in human primary lung cancer tissue using the self-prepared polyclonal antibodies, while it can be detected in cytoplasm of normal lung tissues. The similar result was also testified in A549 cells transfected with or without FUS1 constructs. These results are consistent with previous findings and provide further evidence that FUS1 plays important roles in tumor-suppression function and lung cancer development.

\section{ACKNOWLEDGEMENTS}

This work was supported by the grant from the National Key Research Program for New Drug Development (2009ZX09301-004).

\section{REFERENCES}

[1] Jemal, A., Siegel, R., Ward, E., Hao, Y., Xu, J., Murray, T. and Thun, M.J. (2008) Cancer statistics. A Cancer Journal for Clinicians, 58, 71-96.

[2] Kondo, M., Ji, L., Kamibayashi, C., Tomizawa, Y., Randle, D., Sekido, Y., Yokota, J., Kashuba, V., Zabarovsky, E., Kuzmin, I., Lerman, M., Roth, J. and Minna, J.D. (2001) Overexpression of candidate tumor suppressor gene FUS1 isolated from the 3p21.3 homozygous deletion region leads to G1 arrest and growth inhibition of lung cancer cells. Oncogene, 20, 6258-6262.

[3] Lerman, M.I., Glenn, G.M., Daniel, L., Latif, F., Hosoe, S., Brauch, H., Hampsch, K., Delisio, J., Orcutt, M. and Zbar, B. (1990) A new polymorphic probe on chromosome 3p: Lambda LIB28-77 (D3S169E). Nucleic Acids Research, 18, 205.
[4] Lerman, M.I. and Minna, J.D. (2000) The 630-kb lung cancer homozygous deletion region on human chromosome 3p21.3: Identification and evaluation of the resident candidate tumor suppressor genes. The International Lung Cancer Chromosome 3p21.3 Tumor Suppressor Gene Consortium. Cancer Research, 60, 6116-6133.

[5] Zabarovsky, E.R., Lerman, M.I. and Minna, J.D. (2002) Tumor suppressor genes on chromosome 3p involved in the pathogenesis of lung and other cancers. Oncogene, 21, 69156935.

[6] Uno, F., Sasaki, J., Nishizaki, M., Carboni, G., Xu, K., Atkinson, E.N., Kondo, M., Minna, J.D., Roth, J.A. and Ji, L. (2004) Myristoylation of the FUS1 protein is required for tumor suppression in human lung cancer cells. Cancer Research, 64, 2969-2976.

[7] Prudkin, L., Behrens, C., Liu, D.D., Zhou, X., Ozburn, N.C., Bekele, B.N., Minna, J.D., Moran, C., Roth, J.A., Ji, L. and Wistuba, L.L. (2008) Loss and reduction of FUS1 protein expression is a frequent phenomenon in the pathogenesis of lung cancer. Clinical Cancer Research, 14, 41-47.

[8] Ito, I., Ji, L., Tanaka, F., Saito, Y., Gopalan, B., Branch, C.D., Xu, K., Atkinson, E.N., Bekele, B.N., Stephens, L.C., Minna, J.D., Roth, J.A. and Ramesh, R. (2004) Liposomal vector mediated delivery of the 3p FUS1 gene demonstrates potent antitumor activity against human lung cancer in vivo. Cancer Gene Therapy, 11, 733-739.

[9] Deng, W.G., Kawashima, H., Wu, G., Jayachandran, G., Xu, K., Minna, J.D., Roth, J.A. and Ji, L. (2007) Synergistic tumor suppression by coexpression of FUS1 and p53 is associated with down-regulation of murine double minute-2 and activation of the apoptotic protease-activating factor 1-dependent apoptotic pathway in human nonsmall cell lung cancer cells. Cancer Research, 67, 709-717.

[10] Tsukamoto, H., Fukudome, K., Kohara, J., Nakatake, H. and Kimoto, M. (2007) Preparation of recombinant murine tumor necrosis factor-alpha in Escherichia coli: A rapid method to remove tags from fusion proteins by thrombin-cleavage and ion-exchange chromatography. Protein Expression and Purification, 56, 138-144.

[11] Bradford, M.M. (1976) A rapid and sensitive method for the quantitation of microgram quantities of protein utilizing the principle of protein-dye binding. Analytical Biochemistry, 72, 248-254.

[12] Zhao, X.Y., Li, H.X., Liang, S.F., Yuan, Z., Yan, F., Ruan, X.Z., You, J., Xiong, S.Q., Tang, M.H. and Wei, Y.Q. (2008) Soluble expression of human DRR1 (down-regulated in renal cell carcinoma 1) in Escherichia coli and preparation of its polyclonal antibodies. Biotechnology and Appied Biochemistry, 49, 17-23.

[13] Benzinger, A., Muster, N., Koch, H.B., Yates, J.R. and Hermeking, H. (2005) Targeted proteomic analysis of 14-3-3 sigma, a p53 effector commonly silenced in cancer. Molecular \& Cellular Proteomics, 4, 785-795.

[14] Wilkinson, R.J., Elliott, P., Carragher, J.F. and Francis, G. (2004) Expression, purification, and in vitro characterization of recombinant salmon insulin-like growth factor-II. Protein Expression and Purification, 35, 334-343.

[15] Ji, L., Nishizaki, M., Gao, B., Burbee, D., Kondo, M., Kamibayashi, C., Xu, K., Yen, N., Atkinson, E.N., Fang, B., Lerman, M.I., Roth, J.A. and Minna, J.D. (2002) Ex- 
pression of several genes in the human chromosome 3p21.3 homozygous deletion region by an adenovirus vector results in tumor suppressor activities in vitro and in vivo. Cancer Research, 62, 2715-2720. 